\title{
Tricotilomanía de nueva aparición durante el tratamiento con fármacos estimulantes. A propósito de dos casos clínicos pediátricos
}

\author{
New-onset trichotillomania during treatment with stimulant drugs. About two \\ pediatric clinical cases
}

\author{
Lic. Beatriz Fernández Manso ${ }^{a}$, Lic. Nuria Villora Morcillo ${ }^{a}$ Lic. María A. Taboas Pereira ${ }^{a}$ y \\ Lic. Cristina Elipe Maldonado ${ }^{a}$
}

\begin{abstract}
RESUMEN
Los fármacos estimulantes se usan, habitualmente, en la población pediátrica para tratar el trastorno por déficit de atención e hiperactividad, y sus efectos secundarios están bien descritos. Sin embargo, la tricotilomanía no aparece como uno de ellos. En la literatura, hay algunos casos publicados de tricotilomanía en relación conla administración de metilfenidato y dextroanfetamina. Se presentan dos casos de tricotilomanía de nueva aparición en niños en seguimiento en nuestro Centro por déficit de atención e hiperactividad y en tratamiento con fármacos psicoestimulantes (metilfenidato y lisdexanfetamina), como probable efecto adverso de estos.

Palabras clave: trastorno por déficit de atención con hiperactividad, estimulantes del sistema nervioso central, tricotilomanía, pediatría.
\end{abstract}

\begin{abstract}
Stimulant drugs are commonly used in pediatric population in the treatment of attention deficit hyperactivity disorder, and their side effects are well described, however trichotillomania does not appear as one of them. In the literature we found some published cases of trichotillomania in relation to methylphenidate and dextroamphetamine. We present two cases of new-onset trichotillomania in children followed up in our center by attention deficit hyperactivity disorder and treated with psychostimulant drugs (methylphenidate and lisdexamfetamine), as a probable adverse effect of this treatment. Key words: attention deficit disorder with hyperactivity, central nervous system stimulants, trichotillomania, pediatrics.
\end{abstract}

http: / / dx.doi.org/10.5546/ aap.2020.e61

Cómo citar: Fernández Manso B, Villora Morcillo N, Taboas Pereira MA, Elipe Maldonado C. Tricotilomanía de nueva aparición durante el tratamiento con fármacos estimulantes. A propósito de dos casos clínicos pediátricos. Arch Argent Pediatr 2020;118(1):e61-e62.

a. Hospital Universitario de Fuenlabrada, Fuenlabrada, Madrid, España.

Correspondencia:

Lic. Beatriz Fernández Manso: bfmanso_21@hotmail.com

Financiamiento: Ninguno.

Conflicto de intereses: Ninguno que declarar.

Recibido: 13-2-2019

Aceptado: 6-8-2019

\section{INTRODUCCIÓN}

El uso de fármacos estimulantes, como metilfenidato y lisdexanfetamina, es muy común en el tratamiento del trastorno por déficit de atención e hiperactividad (TDAH). Sus efectos secundarios más habituales son la hiporexia, los trastornos del sueño, el dolor abdominal transitorio y síntomas de nerviosismo o ansiedad. ${ }^{1}$ En la literatura, hay algunas publicaciones que describen, asimismo, la aparición de tricotilomanía en pacientes con este tipo de tratamientos, por lo que existe la posibilidad de que se trate de un efecto adverso inusual no descrito previamente.

Se presentan dos casos, atendidos en nuestro Centro, de tricotilomanía de nueva aparición en niños con TDAH tras el inicio del tratamiento estimulante, como posible efecto secundario inusual de dicha medicación. Ambos fueron notificados al sistema de farmacovigilancia nacional español.

\section{CASOS CLÍNICOS}

Caso 1. Varón de 8 años sin antecedentes de tricotilomanía, con diagnóstico de TDAH a los 6 años, que inició el tratamiento con metilfenidato de acción intermedia a razón de $20 \mathrm{mg} /$ día y asoció, tras su inicio, un episodio autolimitado de tricotilomanía en el cuero cabelludo. Después de 4 meses de tratamiento, presentó de nuevo tricotilomanía en las pestañas y en el cuero cabelludo en relación con un factor estresante, que se resolvió con una disminución de la dosis del fármaco a $10 \mathrm{mg} /$ día. A los 3 meses, se decidió la sustitución por el escaso control de los síntomas nucleares por lisdexanfetamina dismesilato y se reinició la tricotilomanía a la semana del cambio de tratamiento. Por este motivo, se cambió de nuevo a atomoxetina hasta $40 \mathrm{mg} /$ día y, posteriormente, a guanfacina a razón de $3 \mathrm{mg} /$ día para optimizar el control de los síntomas de TDAH, sin presentar nuevos episodios de tricotilomanía. 
Caso 2. Varón sin antecedentes de tricotilomanía con diagnóstico de TDAH a los 8 años sin tratamiento por rechazo de los progenitores. A los 15 años, se decidió, de forma consensuada, el inicio del tratamiento con metilfenidato de acción corta, que, al mes, se cambió por metilfenidato de acción prolongada por mecanismo osmótico hasta $27 \mathrm{mg} /$ día. A las 2 semanas del cambio, presentó un episodio autolimitado de tricotilomanía en las pestañas y, al año, un nuevo episodio similar, que cedió tras la suspensión de la medicación. A los 3 meses, se reinició la medicación con un análogo de acción intermedia de $30 \mathrm{mg}$ / día, y presentó, al mes, de nuevo tricotilomanía en las pestañas, que se resolvió tras la suspensión del fármaco. Un año más tarde, se reinició dicha medicación sin incidencias y fue sustituida, por el escaso control de la sintomatología de TDAH, por lisdexanfetamina con un aumento de dosis gradual. Al alcanzar la dosis de $50 \mathrm{mg} /$ día, reapareció la tricotilomanía en las pestañas, que se resolvió de manera espontánea en corto plazo.

\section{DISCUSIÓN}

Se define la tricotilomanía como el comportamiento repetitivo y recurrente que consiste en el arrancamiento del cabello, asociado a intentos para disminuir o detener dicho comportamiento. Se engloba en la categoría "trastorno obsesivo-compulsivo (TOC) y relacionados" de la clasificación $D S M-5 .{ }^{2}$ Aunque existen estudios que demuestran comorbilidad entre TDAH y conductas obsesivas, como la tricotilomanía, ${ }^{3}$ que pueden exacerbarse con el uso de medicación estimulante, ${ }^{4}$ llama la atención de los casos presentados el comienzo de la sintomatología en relación con el inicio de la medicación estimulante, ya que ninguno de los pacientes había presentado episodios similares previamente a la administración del tratamiento. Además, en ambos casos, existió una relación entre la mejoría o el cese de los síntomas y la suspensión o la bajada de la dosis del medicamento, y estos no reaparecieron con el cambio a otros fármacos no estimulantes, como atomoxetina o guanfacina. Todo ello hace sospechar que la tricotilomanía pueda tratarse de un efecto adverso no conocido de dichos tratamientos.

En la literatura, hay algunos casos similares en niños con TDAH. En 1998, Martin et al., ${ }^{5}$ publicaron el caso de 3 varones de entre 7 y 12 años que presentaron tricotilomanía en un intervalo de 1-6 meses desde el inicio del tratamiento con metilfenidato. En 2013, Narine et al., ${ }^{1}$ reportaron el caso de una adolescente de 12 años que inició la tricotilomanía al comenzar el tratamiento con dextroanfetamina y que se resolvió tras la retirada de la medicación. Similares son los casos publicados en 2017 de dos niños de 8 y 9 años. El primero, ${ }^{6}$ también con diagnóstico de trastorno del espectro autista, presentó tricotilomanía al mes de haber iniciado el tratamiento con metilfenidato, $\mathrm{y}$, aunque los síntomas se resolvieron tras su suspensión, reaparecieron tras 2 semanas desde el reinicio del tratamiento. El segundo ${ }^{7}$ presentó los síntomas a la semana de haber iniciado el tratamiento con metilfenidato de liberación prolongada y se resolvieron tras su retirada, sin volver a presentarlos al iniciar el tratamiento con atomoxetina. De acuerdo con nuestro conocimiento, no se han reportado previamente casos en la literatura de tricotilomanía durante el tratamiento con lisdexanfetamina en la edad pediátrica, como sucedió en nuestros casos.

Los psicoestimulantes aumentan la concentración de dopamina, que, junto con la serotonina, es uno de los neurotransmisores implicados en la etiología de las conductas compulsivas y, en concreto, de la tricotilomanía. Por tanto, la asociación entre tricotilomanía y TDAH podría tratarse de una complicación del tratamiento estimulante, o bien, la expresión de una vulnerabilidad neurobiológica que se encontraba latente. ${ }^{2}$ Se concluye que son necesarios nuevos estudios para esclarecer la posible relación entre la tricotilomanía y el tratamiento con fármacos psicoestimulantes.

\section{REFERENCIAS}

1. NarineC,SarwarS,Rais T.Adderall-induced Trichotillomania: a case report. Innov Clin Neurosci. 2013; 10(7-8):13-4.

2. American Psychiatric Association. DSM-5: Manual Diagnóstico y Estadístico de los Trastornos Mentales. $5^{\mathrm{a}}$ ed. Madrid: Panamericana; 2014.

3. Abramovitch A, Dar R, Mittelman A, Wilhelm S. Comorbidity Between Attention Deficit/Hyperactivity Disorder and Obsessive-Compulsive Disorder Across the Lifespan: A Systematic and Critical Review. Harv Rev Psychiatry. 2015; 23(4):245-62.

4. Borcherding BG, Keyson CS, Rapaport JL, Elia J, et al. Motor/vocal tics and compulsive behaviors on stimulant drugs: Is there a common vulnerability? Psychiatry Res. 1990; 33(1):83-94.

5. Martin A, Scahill L, Vitulano L, King RA. Stimulant use and trichotillomania. J Am Acad Child Adolesc Psychiatry. 1998; 37(4):349-50.

6. Gunes S. Modified-Release Methylphenidate-Related Trichotillomania in a Boy with Autism Spectrum Disorder. J Child Adolesc Psycopharmacol. 2017; 27(7):675-6.

7. Kara T, Akaltum I. Trichotillomania in Attention-Deficit/ Hyperactivity DisorderUnder Methylphenidate Treatment. J Clin Psycopharmacol. 2017; 37(4):484. 